\title{
A positive view on e-cigarettes
}

\author{
Jamie Brown SSA (Society for Study of Addiction) senior research fellow \\ University College London, London WC1E 6BT, UK
}

Gornall's representation of the evidence on e-cigarettes is fairly one sided and negative. ${ }^{1}$ For example, he cites a "recent meta-analysis" on the effectiveness of e-cigarettes, which was posted on a blog and has not been peer-reviewed ${ }^{2}$ but fails to cite the Cochrane review on the same topic, which was published just three months earlier. ${ }^{3}$ While noting that the quality of evidence is low, the Cochrane review came to different conclusions: e-cigarettes help smokers to stop long term compared with placebo e-cigarettes. There was uncertainty about the relative effect compared with nicotine patches on quitting, but people who used e-cigarettes compared with patches were more likely to cut cigarette consumption by at least half. There was no evidence of an association between short term use and health risks. The Cochrane Library is widely recognised as a gold standard repository for high quality, independent evidence to inform healthcare decision making. I am not sure that the blog cited is held in the same regard.

Gornall's feature could not provide an exhaustive list of all organisations and their positions on e-cigarettes. ${ }^{1}$ However, given the unfair implication that certain organisations have been acting somewhat partially, it is important to note that prominent UK health organisations other than those cited have also taken a comparatively positive view of the evidence on e-cigarettes. For example, the National Centre for Smoking Cessation and Training produced a briefing in partnership with Public Health England that recommends practitioners "be open to e-cigarette use in people keen to try them; especially in those that have tried, but not succeeded, in stopping smoking with the use of licensed stop smoking medicines. ${ }^{44}$ And a recent blog post by
Clive Bates (a former director of ASH) provides a summary indication of the positive view of several other organisations by listing their positions towards the ban on indoor vaping in Wales. ${ }^{5}$ These organisations included the Royal College of Physicians (Wales), which "anticipates that e-cigarettes and related products could actually generate significant falls in the prevalence of smoking in the UK, prevent many deaths and episodes of serious illness, and help to reduce social inequalities in health." ${ }^{6}$

Competing interests: JB is funded by a fellowship from the UK Society for the Study of Addiction. He has received unrestricted research funding from Pfizer but does not, and will not, take research funding from electronic cigarettes manufacturers or the tobacco industry. Full response at: www.bmj.com/content/350/bmj.h3317/rr-0.

1 Gornall J. Why e-cigarettes are dividing the public health community. BMJ 2015;350:h3317. (24 June.)

2 Glantz S. Meta-analysis of all available population studies continues to show smokers who use e-cigs less likely to quit smoking. 2015. http://tobacco.ucsf.edu/meta-analysisall-available-population-studies-continues-show-smokers-who-use-e-cigs-less-likely-quitsmoking.

3 McRobbie H, Bullen C, Hartmann-Boyce J, et al. Electronic cigarettes for smoking cessation and reduction. Cochrane Database Syst Rev 2014;12:CD010216.

4 National Centre for Smoking Cessation and Training (NCSCT). Electronic cigarettes. 2014. www.ncsct.co.uk/usr/pub/e-cigarette_briefing.pdf.

5 Bates $C$. Wales vaping ban: silver lining may be larger than cloud. 2015. www.clivebates. $\mathrm{com} / \mathrm{p}=3096$

6 Royal College Physicians (Wales). Reducing health inequalities: RCP (Wales) policy response. 2014. www.rcplondon.ac.uk/sites/default/files/rcp_wales_response_to_ consultation_on_reducing_health_inequalities.pdf. 